\title{
¿Cómo evitar lesiones osteomusculares durante la realización de ecocardiogramas?
}

\author{
Paula Andrea Cuartas González \\ Hospital Universitario Mayor-MÉDERI. Bogotá DC-Colombia \\ Recibido: $31 / 05 / 2020$ \\ Aceptado: 13/10/2020 \\ En línea: 31/12/2020
}

Correspondencia

Paula Andrea Cuartas González.

pauliscuartis@gmail.com

Citar como: Cuartas González P. A. ¿Cómo evitar lesiones osteomusculares durante la realización de ecocardiogramas? Rev Ecocar Pract (RETIC). 2020 (Dic); 3 (3): 48-51. doi: 10.37615/retic.v3n3a4.

Cite this as: Cuartas González P. A. How to avoid musculoskeletal injuries during echocardiograms performance? Rev Ecocar Pract (RETIC). 2020 (Dic); 3 (3): $48-51$. doi: 10.37615/retic.v3n3a4

\section{Palabras clave \\ $\triangleright$ Ergonomía \\ $\triangleright$ Lesiones osteomusculares \\ $\triangleright$ Sonografistas cardíacos \\ $\triangleright$ Ecocardiografía}

\begin{tabular}{l}
\hline Keywords \\
\hline$\triangleright$ Ergonomics \\
$\triangleright$ Musculoskeletal injuries \\
$\triangleright$ Cardiac sonographers \\
$\triangleright$ Echocardiography
\end{tabular}

\begin{abstract}
RESUMEN
Las lesiones osteomusculares en profesionales que realizan ecocardiogramas son problemas de causa multifactorial y alta incidencia. También es frecuente la presencia de dolor que dificulta la realización de las actividades laborales. Ambas condiciones generan incapacidad y deterioran la calidad de vida de estos profesionales. La identificación precoz de estos problemas y la implementación de medidas de prevención ayudarán a mejorar la productividad y satisfacción laboral.
\end{abstract}

ABSTRACT
Osteomuscular injuries in professionals who perform echocardiograms are common problems of multifactorial
cause. The presence of pain that makes it difficult to carry out work activities is also frequent. Both conditions ge-
nerate disability and deteriorate the quality of life of these professionals. The early identification of these problems
and the implementation of prevention measures will help to improve productivity and job satisfaction.

\section{Definición de lesiones osteomusculares en profesionales que realizan exámenes de ultrasonido}

Son lesiones en trabajadores cuya ocupación requiere la repetición de maniobras isométricas, mala postura, estrés o presión constante sobre grupos musculares específicos durante el trabajo repetitivo, y que se traduce en artralgias y dolor muscular.

Se ha estudiado su presentación en diferentes especialidades como obstetricia, medicina general, cardiovascular y osteomuscular, demostrándose que el mayor riesgo de lesión lo tienen los profesionales de la salud que se dedican al ultrasonido cardíaco (1).

\section{Tipo de lesiones osteomusculares}

Las lesiones osteomusculares más frecuentemente descritas son (2):

- Mano: síndrome de túnel del carpo, tendinitis, tenosinovitis de la mano (como el síndrome de Quervain) y la muñeca, dedos en gatillo.

- Hombro: bursitis, tendinitis y enfermedad del manguito rotador.

- Brazo: síndrome cubital.

- Caja torácica: síndrome de abertura torácica superior (compresión de los vasos sanguíneos o terminaciones nerviosas en el espacio entre la clavícula y la primera costilla).

- Columna: escoliosis, hernia discal.

- Cadera: compresión del nervio ciático, alteración de la articulación de la cadera y la rodilla.

\section{Signos y síntomas}

La presencia de lesiones osteomusculares se asocia a signos y síntomas como: dolor, hinchazón, calor local, entumecimiento, hormigueo, espasmos musculares, pérdida de la sensibilidad, pérdida de la fuerza muscular y cansancio generalizado.

Se ha identificado la presencia de dolor durante la realización de las actividades laborales, comprometiendo inclusive la realización de actividades en el hogar, afectando la calidad de vida del profesional de la salud e incrementando los días de incapacidad. El dolor y pérdida de la fuerza muscular suelen aparecer en el lado del cuerpo que el profesional utiliza para escanear (incluso puede dificultar sostener el transductor), aunque también puede sentir dolor en la extremidad que utiliza para la operación de los controles del ecógrafo. Se ha descrito frecuentemente la presencia de dolor en: hombros, cuello, espalda alta y baja, muñeca, manos y dedos, escápula, antebrazo y codos.

\section{Factores de riesgo \\ para desarrollar lesiones}

Las lesiones osteomusculares son multifactoriales, su aparición se puede asociar a la presencia de varios factores que ejercen sinergia hasta generar la lesión.

Entre los más frecuentemente descritos se encuentran:

- Factores ergonómicos: relacionados con las posturas y factores biomecánicos durante el trabajo. 
- Factores psicosociales: relacionados con el individuo y sus dinámicas laborales.

- Factores administrativos: relacionados con la gestión y administración de los centros de diagnóstico, la productividad, costo-efectividad, entre otros. El aumento en el número de exámenes conlleva a un menor tiempo de recuperación muscular.

La tendencia de hacer más exámenes es servicios fuera de la sala de ultrasonografía (centro quirúrgico, cuidados intensivos, cuidados coronarios), constituye un desafío ergonómico debido a condiciones técnicas inadecuadas.

\section{¿Qué condiciones conllevan}

a lesiones osteomusculares?

- El uso masivo del ultrasonido cardíaco, con aumento del número de pacientes por día, de evaluaciones por paciente y aumento de la complejidad de los exámenes.

- La realización de exámenes en la cama del paciente, con necesidad de desplazar equipos pesados y condiciones técnicas (posición e iluminación) inadecuadas para la realización de los estudios.

- El desarrollo tecnológico de los ecógrafos (que hace que el proceso de adquisición necesite múltiples vistas y modos de escaneo, diferentes tipos de transductores, uso de protocolos extensos para obtener imágenes), aumenta el tiempo de adquisición y procesamiento de los exámenes.

- El aumento en el número y la duración de los turnos de trabajo, compuestos por jornadas continuas y extensas.

- La excesiva presión aplicada al transductor, especialmente en pacientes con obesidad, patologías respiratorias y ventilación invasiva.

- El uso de posturas inadecuadas (mala relación entre la posición del ecógrafo, la camilla y el operador), movimientos repetitivos, movimientos torpes durante el entrenamiento, todos pueden ocasionar lesiones osteomusculares lo cual se traduce en cansancio muscular y trauma acumulativo (ver Figuras 1-6).

\section{¿Cómo prevenir}

las lesiones osteomusculares?

La prevención de las lesiones osteomusculares se basa en la intervención precoz de los principales factores desencadenantes ${ }^{(3)}$ (Figura 7, Figura 8 y Figura 9):

- Optimización de la postura en los exámenes en la cama del paciente y en el consultorio: ubicar el equipo al frente del operador, la silla y cama de examen a la altura del operador para evitar el estiramiento y abducción excesiva del brazo y hombro.

El monitor del equipo ubicado a la altura de los ojos permite una posición neutral del cuello. Ubicar el paciente lo más cercano posible al operador, disminuye el ángulo de abducción del hombro y el brazo y evita el estiramiento excesivo

- Uso de dispositivos de soporte: estos dispositivos brindan soporte al codo y antebrazo, disminuyen el ángulo de abducción del hombro y liberan el peso del cable del transductor de la mano del operador.

- Uso de pausas activas, hacer ejercicios de calentamiento y estiramiento, establecer un número límite de procedimientos por día y evitar jornadas continuas y extenuantes de trabajo.

- Hacer una reingeniería del sitio de trabajo teniendo en cuenta las sugerencias de ergonomía e higiene laboral, con el objetivo de optimizar las salas de examen con respecto a su distribución, tamaño, ventilación y adecuada iluminación.

- Adquisición de nuevos ecógrafos de fácil desplazamiento, que se adapten a la presencia de múltiples dispositivos. Los fabricantes han desarrollado diseños que permiten ubicar los ecógrafos en lugares de difícil acceso.

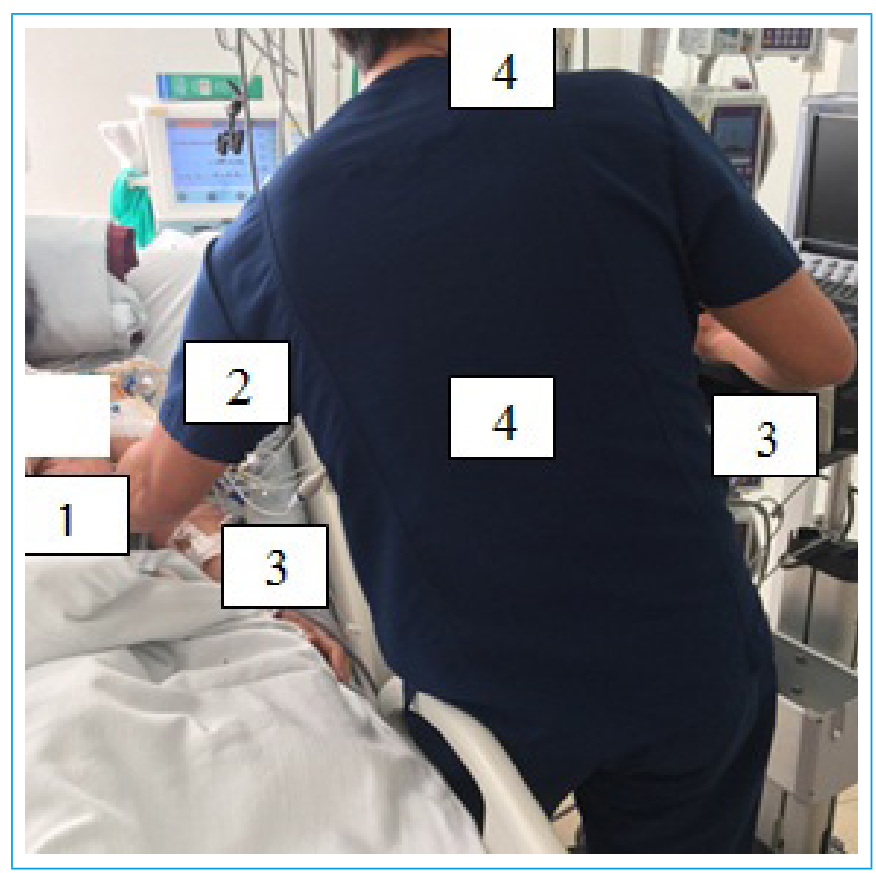

Figura 1. Postura inadecuada de escaneo. Factores biomecánicos y condiciones técnicas complejas, durante un procedimiento en la cama del paciente. Se observa: 1. Brazo y antebrazo sin soporte; 2. Abducción excesiva del hombro mayor a 30 grados; 3 . Extensión inadecuada del brazo mayor a 30 cms; 4. Rotación, extensión y flexión inadecuadas del tronco y el cuello

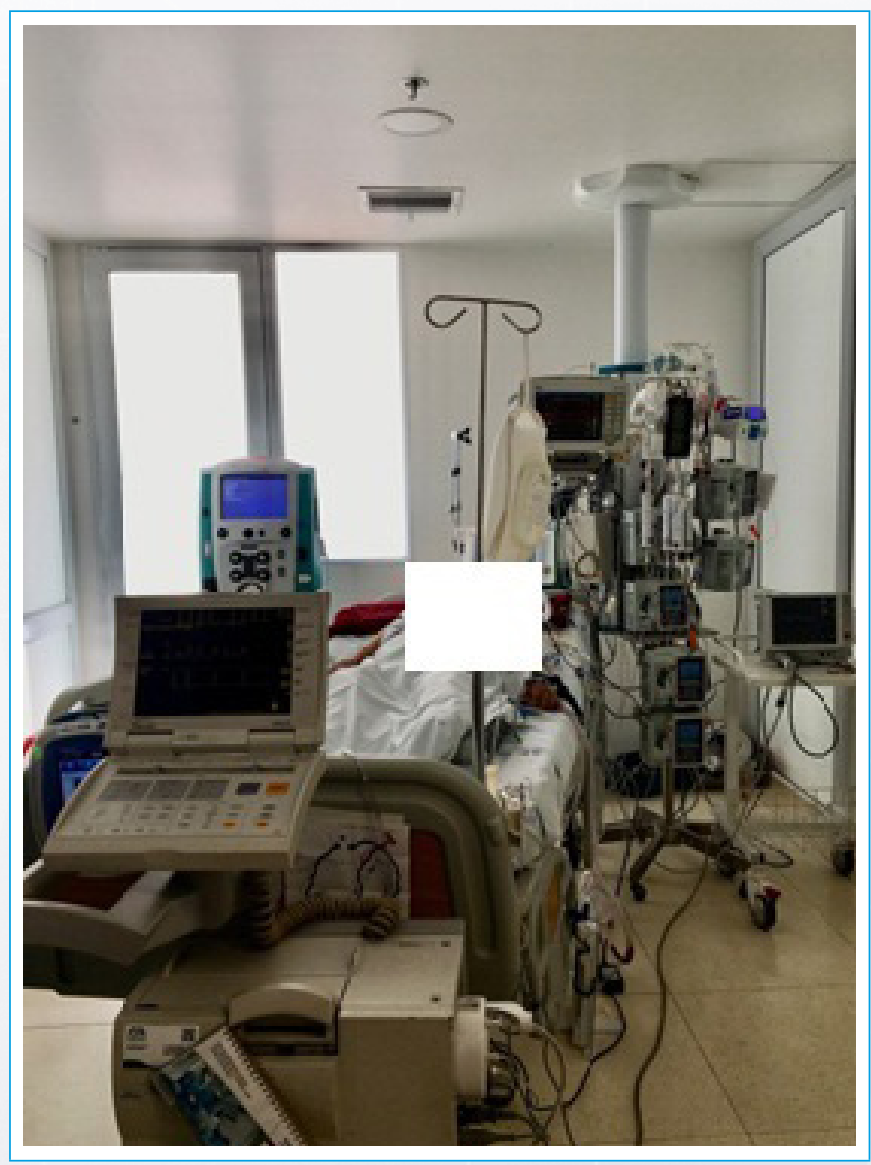

Figura 2. Habitaciones en cuidados intensivos con múltiples dispositivos biomédicos, que deben ubicarse adecuadamente para garantizar una adecuada postura de escaneo 


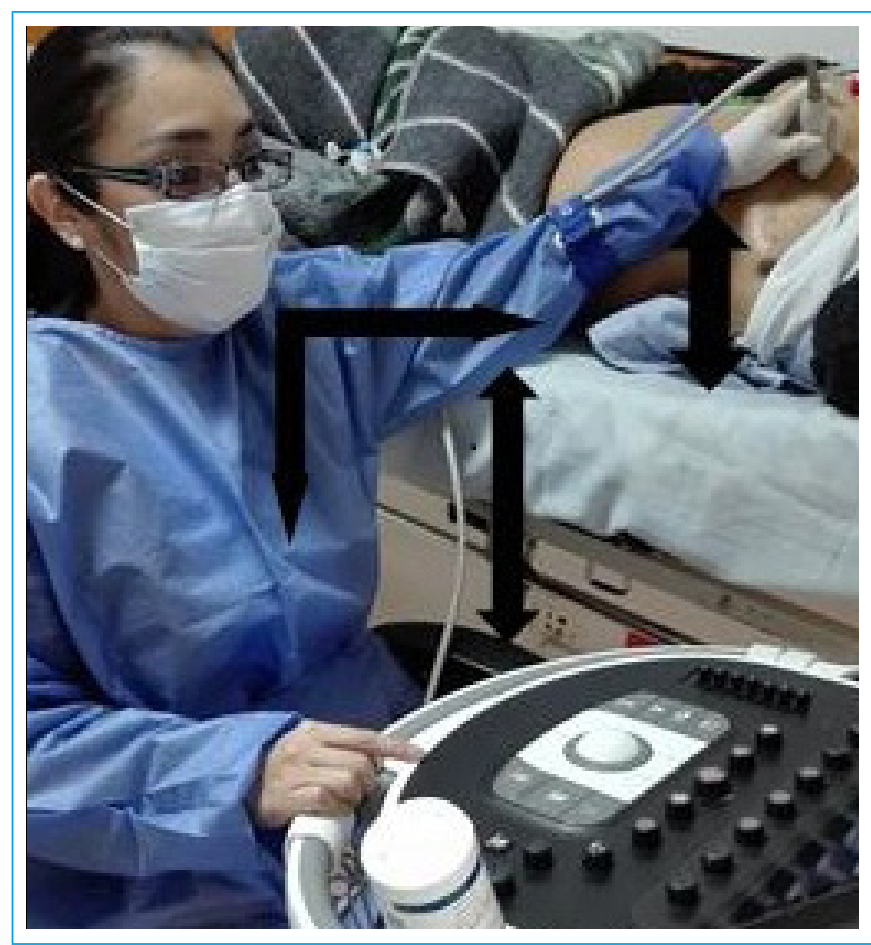

Figura 3. Silla y camilla de examen inadecuados. Factores biomecánicos: En este ejemplo la silla no alcanza a graduarse al tamaño de la camilla; la camilla es rígida y no permite graduar su altura; el operador se encuentra lejos del paciente y de manera compensadora aumenta el ángulo de abducción del hombro y estira el brazo; por la distancia para alcanzar el paciente no es posible utilizar elementos de soporte quedando el codo y el brazo inestables. Es una postura de riesgo para el desarrollo de lesiones osteomusculares y dolor

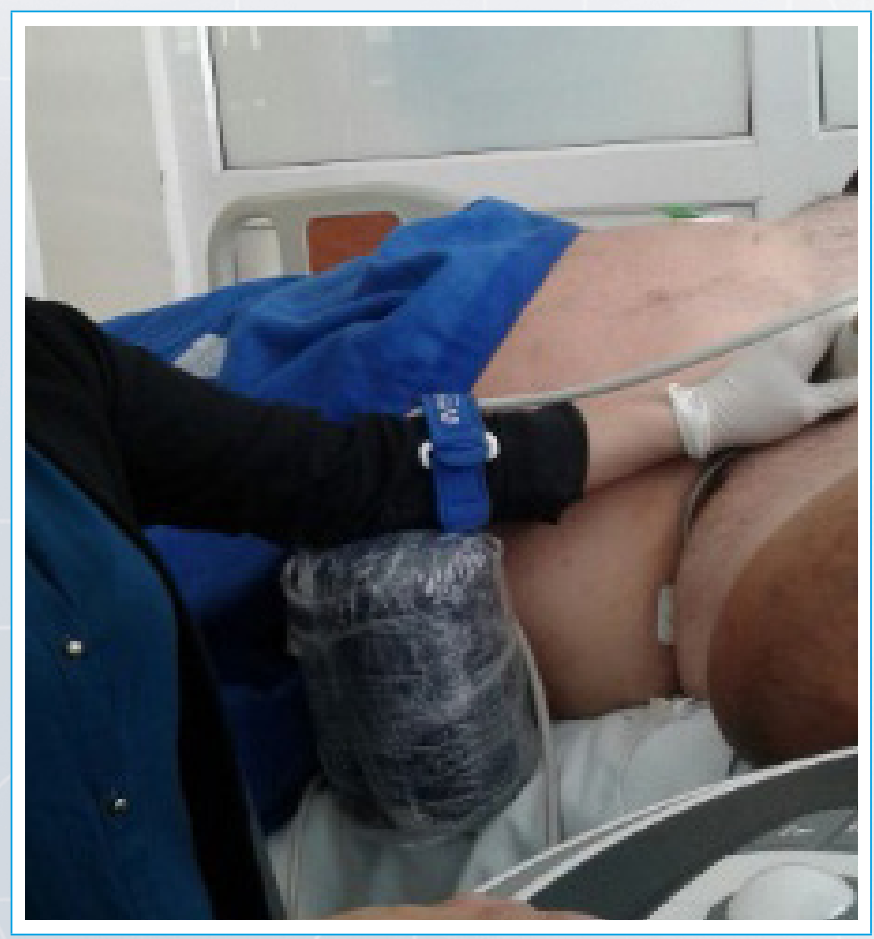

Figura 4. Paciente en posición inadecuada. Factores biomecánicos: En esta imagen se observa que el paciente, por su contextura física, no puede adoptar la posición para el examen, ubicándose lejos del operador y haciendo que este se incline hacia la izquierda, aumente el ángulo de abducción del hombro y flexione el cuello. El uso de dispositivos de soporte permite el apoyo del codo y la muñeca

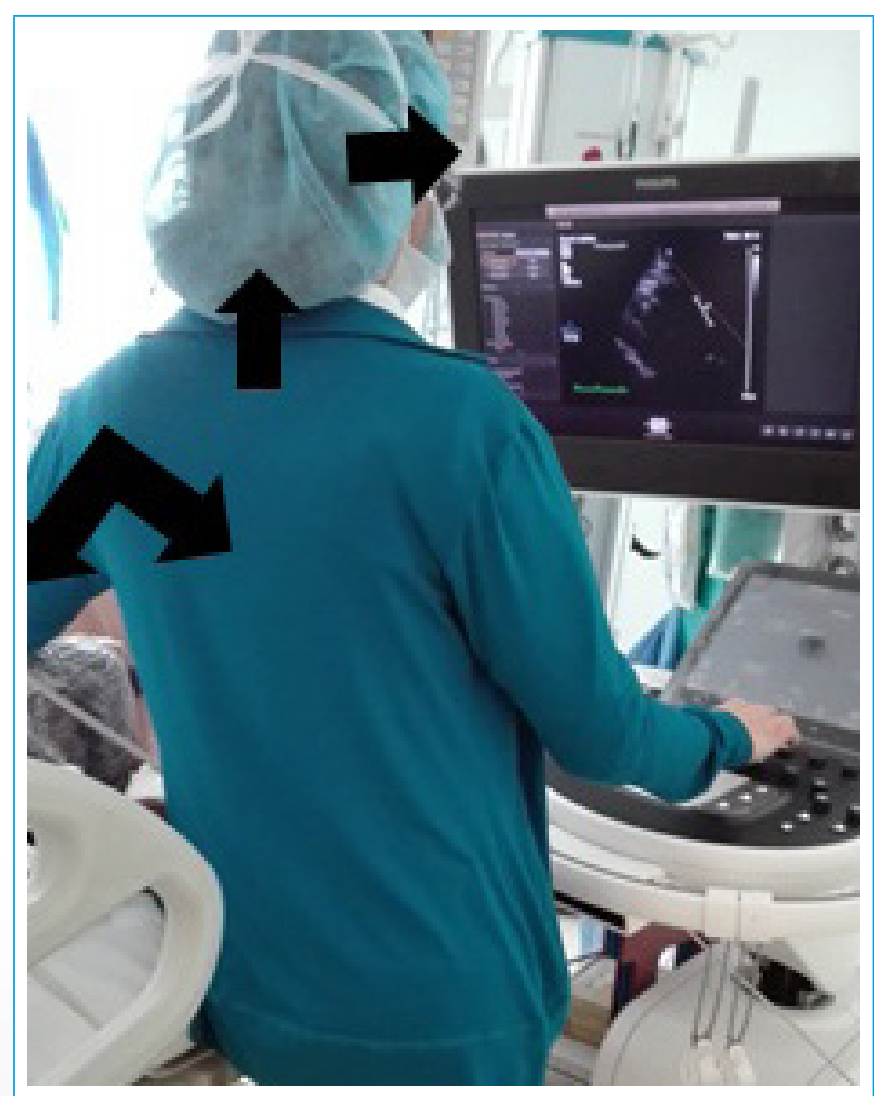

Figura 5. Operador escaneando un paciente de pie. Factores biomecánicos: En esta imagen el operador es visto desde atrás y desde el frente, se observa una inadecuada postura por aumento del ángulo de abducción del hombro y la pantalla del equipo no se ubica a la altura de los ojos, haciendo que el operador baje la mirada y extienda su cuello.

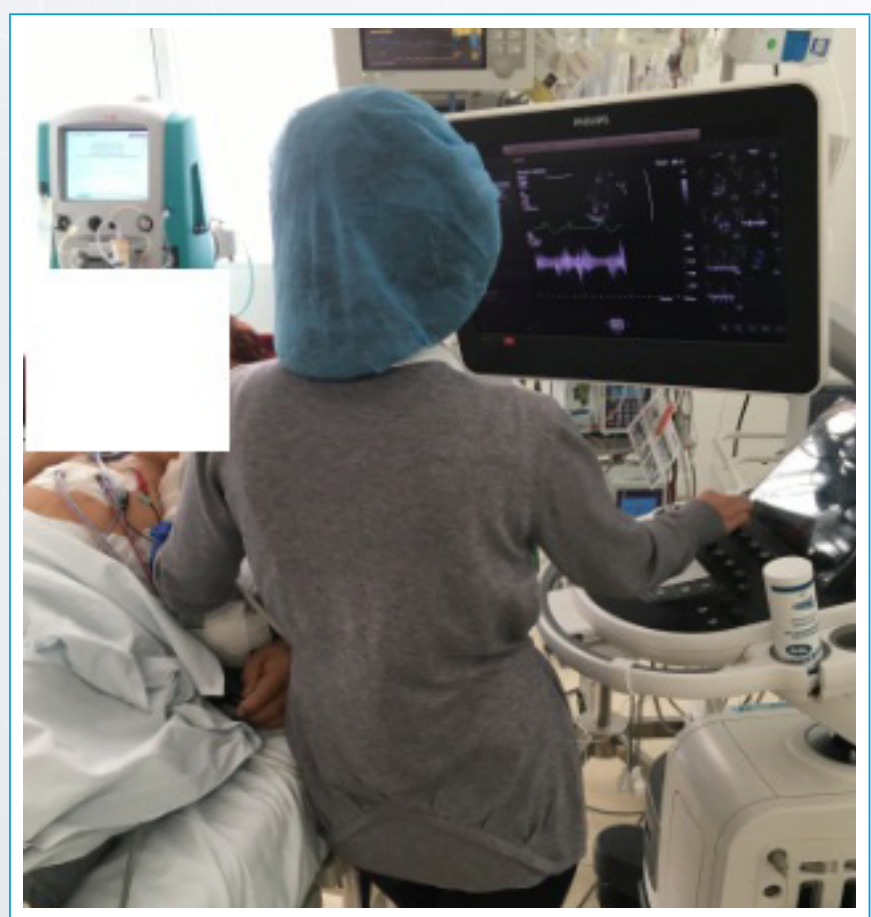

Figura 6. Operador escaneando un paciente de pie. Factores biomecánicos: En esta imagen el operador es visto desde atrás y desde el frente, se observa una inadecuada postura por aumento del ángulo de abducción del hombro y la pantalla del equipo no se ubica a la altura de los ojos, haciendo que el operador baje la mirada y extienda su cuello. 
- Trukipedia

Truco 01

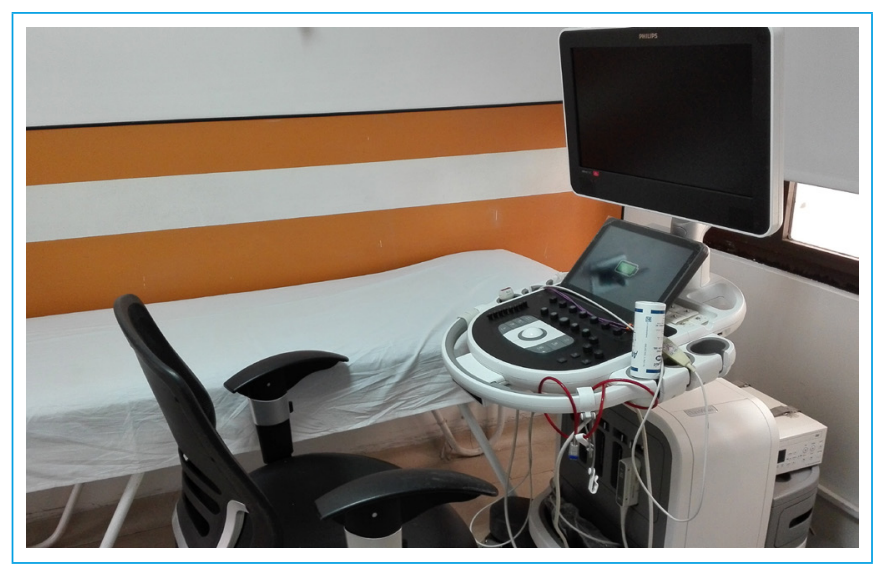

Figura 7. Sala de examen con optimización de la distribución

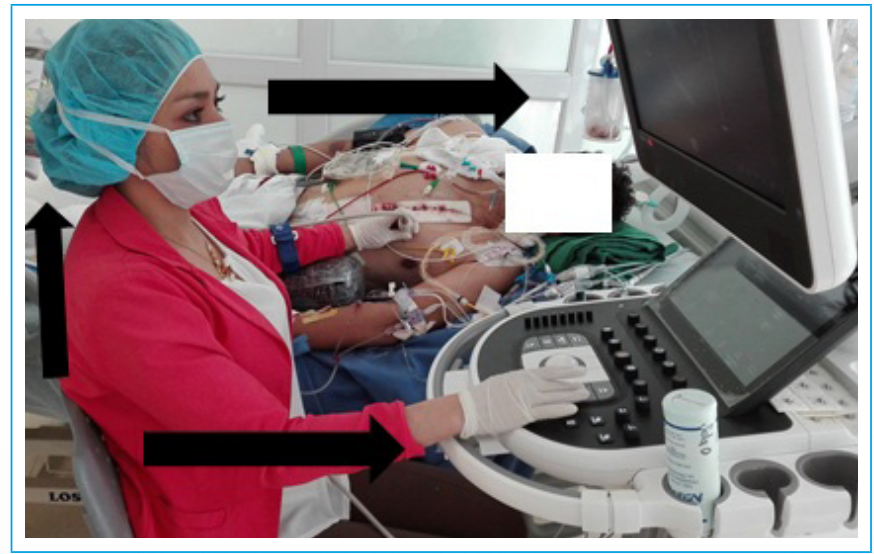

Figura 8. Examen en la cama del paciente con optimización de la postura y uso de dispositivos de soporte

\section{Ideas para recordar}

El conocimiento de las lesiones osteomusculares relacionadas con el uso del ultrasonido diagnóstico permitirá identificar la presencia de factores de riesgo, implementar medidas preventivas y prácticas seguras durante el trabajo, y así evitar lesiones definitivas e incapacidad permanente en los profesionales de la salud..

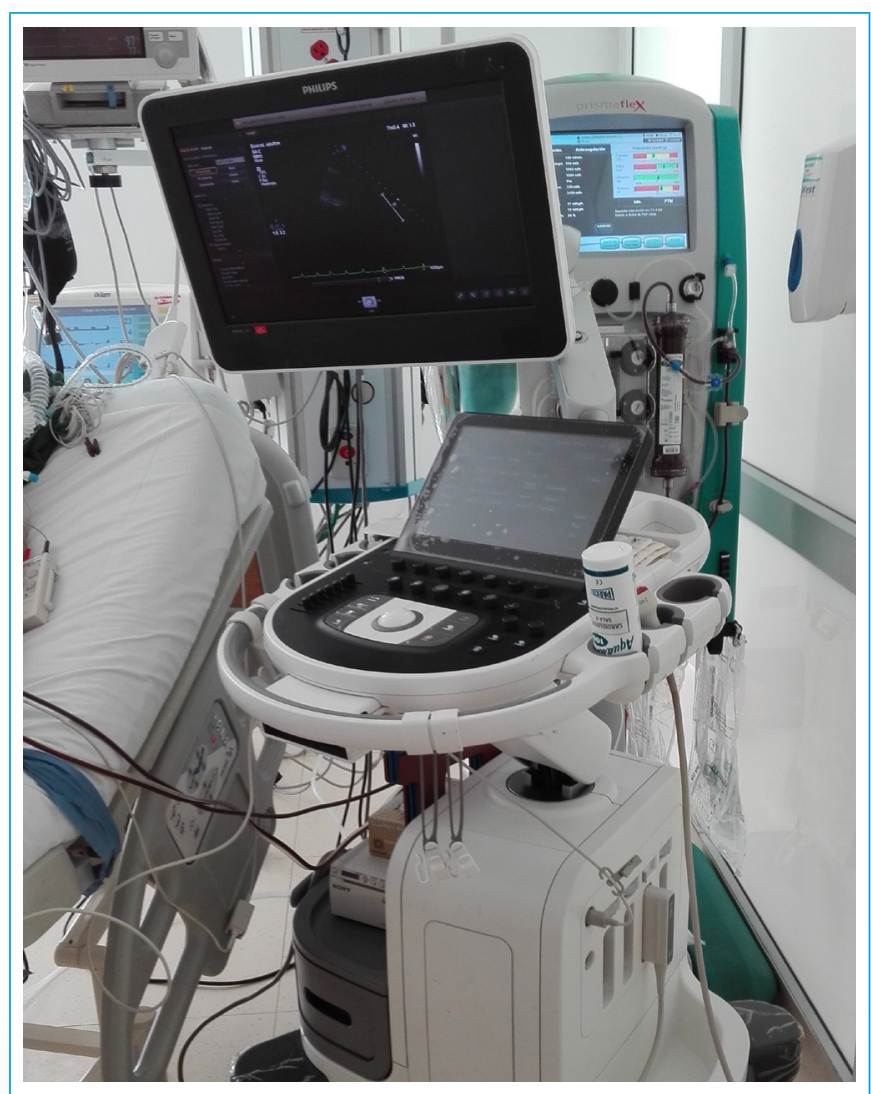

Figura 9. Ecógrafo que se puede replegar. El ecógrafo se permite plegar su estructura, graduar la altura de los controles y de la pantalla

\section{Bibliografía}

1. Zhang D, Huang H. Prevalence of work-related musculoskeletal disorders among sonographers in China: results from a national web-based survey, Journal of Occupational Health 2017; 59 (6): 529-541.

2. Coffin C. Work-related musculoskeletal disorders in sonographers: a review of causes and types of injury and best practices for reducing injury risk. Reports in Medical Imaging 2014;7: 15- 26.

3. Harrison G, Harris A. Work-related musculoskeletal disorders in ultrasound: Can you reduce risk? Ultrasound 2015; 23: 224-230. 